\title{
Transseptal mitral valve-in-valve implantation helps to elucidate the degree of concomitant aortic valve stenosis
} \author{
Davide Gabbieri $^{4}$ \\ ${ }^{1}$ Department of Cardiology, University Hospital in Opole, Opole, Poland \\ ${ }^{2}$ Faculty of Physical Education and Physiotherapy, Opole University of Technology, Opole, Poland \\ ${ }^{3}$ Department of Cardiac Surgery, University Hospital in Opole, Opole,Poland \\ ${ }^{4}$ Department of Cardiovascular Surgery, Hesperia Hospital, Modena, Italy
}

Jerzy Sacha ${ }^{1,2}$, Krzysztof Krawczyk $^{1}$, Paweł Tomaszewski ${ }^{3}$, Jarosław Bugajski ${ }^{1}$, Radosław Gawroński ${ }^{3}$,

Adv Interv Cardiol 2020; 16, 4 (62): 516-518

DOI: https://doi.org/10.5114/aic.2020.101783

Evaluation of the severity of aortic valve stenosis (AVS) in concomitant significant mitral valve regurgitation is challenging. Since the risk of double-valve versus single-valve surgery is remarkably higher, reliable assessment of multivalvular heart disease is crucial, particularly in the case of re-operation [1]. This clinical example demonstrates how a transseptal mitral valve-in-valve implantation may help to elucidate the degree of borderline AVS. In addition, the double wire technique of the valve implantation is presented.

A 77-year-old woman after biological valve implantation in 2006 (Hancock II 29 mm) was admitted due to heart failure symptoms (NYHA III). She also suffered from chronic atrial fibrillation, hypertension, hypercholesterolemia, coxarthrosis and spondylarthrosis, and she experienced a brain stem stroke in 2013. Transthoracic and transesophageal echocardiography revealed good left ventricular function (ejection fraction 57\%), severe mitral prothesis regurgitation due to leaflet perforation (Figure $1 \mathrm{~A})$ (ERO $0.51 \mathrm{~cm}^{2}$, regurgitation volume $80 \mathrm{ml}$, maximal/mean gradient 21/6 mm Hg), moderate functional tricuspid valve regurgitation and borderline AVS (aortic valve area (AVA) calculated from the continuity equation $1.1 \mathrm{~cm}^{2}$ and its index (AVAI) $0.67 \mathrm{~cm}^{2} / \mathrm{m}^{2}$; on planimetry, AVA $0.98 \mathrm{~cm}^{2}$, AVAI 0.59 $\mathrm{cm}^{2} / \mathrm{m}^{2}$; maximal/mean gradient $28 / 14 \mathrm{~mm} \mathrm{Hg}$ ). Since the stroke volume and its index were borderline (SV $60 \mathrm{ml}, \mathrm{SVI} 36 \mathrm{ml} / \mathrm{m}^{2}$, respectively) and the mitral regurgitation volume was high, the severity of AVS could not be determined - i.e., it was possible that after fixing the mitral regurgitation the aortic stenosis would become significant, particularly as the calcium score according to multi-slice computed tomography (MSCT) was high (i.e. 1555) [1].

According to EuroSCORE II and STS, the risk of surgical mortality for mitral valve re-operation was $12.21 \%$ and $7.6 \%$ respectively; however, the risk of a double valve operation was certainly much higher but undetermined. After interdisciplinary discussion, the Heart Team recommended a transcatheter mitral valve-in-valve implantation, then an intra-operative assessment of the aortic valve and a transcatheter aortic valve implantation (TAVI) in the case of significant aortic stenosis. On MSCT, the risk of significant left ventricular outflow tract (LVOT) obstruction was low (the estimated neo-LVOT area was $2.3 \mathrm{~cm}^{2}$ ).

Using a transseptal approach, a 29-mm SAPIEN3 (Edwards Lifesciences) transcatheter valve was implanted with a double wire technique (Figures 1 B-E). In this technique, two small Safari wires (Boston Scientific) are inserted through an interatrial septum and mitral bio-prothesis into the left ventricle. One of the wires serves as a buddy wire and facilitates SAPIEN3 valve delivery - such a wire is helpful if the SAPIEN3 valve is stuck at the septum or at the annulus of the bio-prothesis since it enables a small balloon to be inserted and the valve to be pushed in the right direction [2].

After successful implantation, the invasive and non-invasive evaluation revealed a moderate degree of AVS (AVA $1.1 \mathrm{~cm}^{2}$, maximal/mean gradient 24/11 $\mathrm{mm} \mathrm{Hg}$ ), and hence, TAVI was abandoned. During 6-month follow-up, the patient was asymptomatic with good function of the implanted SAPIEN3 valve and no signs of progression of aortic stenosis. This case shows that

\section{Corresponding author:}

Jerzy Sacha MD, PhD, Department of Cardiology, University Hospital in Opole, Al. Witosa 26, 45-401 Opole, Poland, phone: +48 774520872 , e-mail: sacha@op.pl

Received: 12.07.2020, accepted: 13.09.2020. 

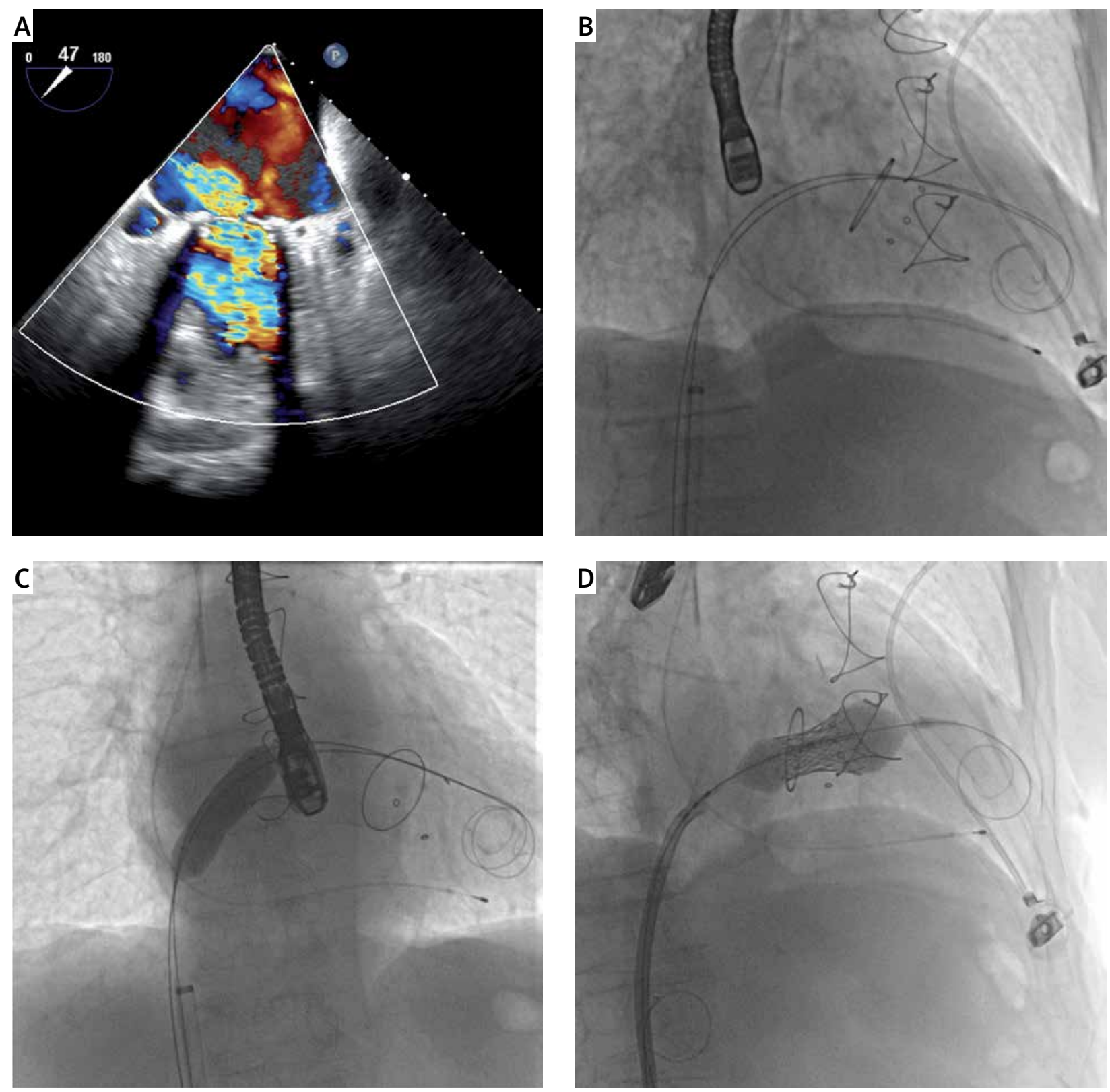

Figure 1. Severe mitral bio-prothesis regurgitation treated with transseptal mitral valve-in-valve implantation and subsequent assessment of borderline aortic stenosis is presented. A - Transesophageal echocardiography shows significant regurgitation of mitral biological prothesis (Hancock II $29 \mathrm{~mm}$ ). B - After transseptal puncture, two small Safari wires were inserted into the left ventricle with the help of an Agilis catheter (Abbott) and 7F JR4 catheter, then an Edwards eSheath introducer was placed over one of the wires (i.e. a working wire) - another wire served as a buddy wire and was kept outside the eSheath. C - Using the working Safari wire, a balloon septostomy was performed with a $14 \mathrm{~mm}$ Valver balloon (Balton). D - The SAPIEN3 valve was delivered, then the buddy wire was removed and the valve was implanted during prolonged fast cardiac pacing 

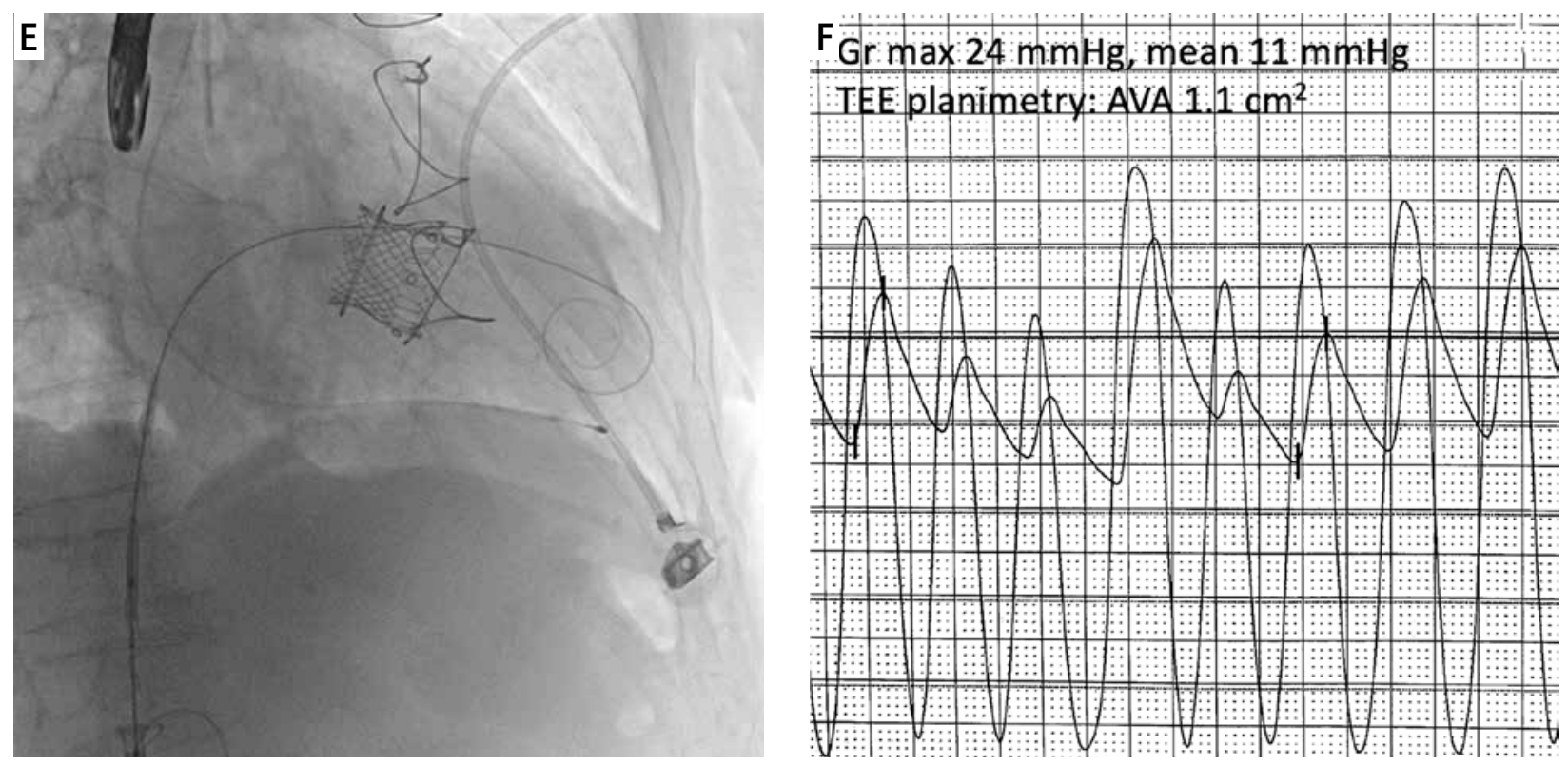

Figure 1. Cont. E - To ensure a conical shape of the implanted valve and prevent its late migration to the left atrium an oversized $29 \mathrm{~mm}$ SAPIEN3 valve was used in this case. $\mathbf{F}$ - Immediately after the procedure, the aortic valve gradient and aortic valve area measurements revealed features of moderate stenosis

Gr-gradient, TEE - transesophageal echocardiography, AVA - aortic valve area.

a transcatheter approach may help to manage multivalvular heart disease in an evidence-based manner.

\section{Conflict of interest}

The authors declare no conflict of interest.

\section{References}

1. Baumgartner H, Falk V, Bax J, et al. 2017 ESC/EACTS Guidelines for the management of valvular heart disease. Eur Heart J 2017; 38: 2739-91.

2. Hachinohe D, Latib A, Mitomo S, et al. Usefulness of buddy wire technique during transcatheter transseptal mitral valve implantation in degenerated mitral bioprosthesis. Circ J 2018; 83: 243-4. 